\title{
Metodologías participativas aplicadas a la socio-hidrología y su potencial para la incidencia social: algunas reflexiones
}

\section{Participative methodologies applied to socio-hydrology and their potential for social change: some reflections}

DOI 10.15517/rr.v99i2.38597

\author{
Édgar Espinoza Cisneros ${ }^{1,2}$ \\ Sara Blanco Ramírez ${ }^{2}$
}

${ }^{1}$ Escuela de Geografía, Universidad de Costa Rica, Costa Rica, edgar.espinoza@ ucr.ac.cr

ORCID - https://orcid.org/0000-0002-1018-3440

${ }^{2}$ Observatorio del Agua y Cambio Global, Universidad de Costa Rica, Costa Rica, sara.blanco@ucr.ac.cr

ORCID - https://orcid.org/0000-0002-9638-6272

Fecha de recepción: 9 de agostos del 2019 Fecha de aceptación: 23 de mayo del 2020

\section{Resumen}

Introducción y objetivo: En el marco de las II Jornadas de Investigación de la Facultad de Ciencias Sociales de la Universidad de Costa Rica (UCR), este artículo examina el potencial de incidencia social de metodologías participativas para la gestión ambiental, especialmente en el campo emergente de la socio-hidrología. Para ello, nos apoyamos en dos proyectos de investigación que actualmente están en desarrollo y que pretenden incidir en la toma de decisiones en diversos niveles de gobernanza.

Método y técnica: Ambos proyectos aplican nuevas tendencias participativas que enfatizan el componente tecnológico. Uno de estos proyectos se vale de la ciencia ciudadana para fortalecer las capacidades de gestión ambiental de grupos comunitarios a través del monitoreo participativo de la calidad del agua superficial en una cuenca en la zona norte de Costa Rica, contexto caracterizado por crecientes afectaciones socio-ecológicas a raíz de actividades agrícolas intensivas, particularmente la contaminación de fuentes de agua. El otro proyecto presentado utiliza sistemas de información geográfica participativos (SIGP) para el mapeo y análisis espacial de valores sociales atribuidos a los servicios ecosistémicos proveídos por los sistemas fluviales en la cuenca del río Savegre, en la vertiente Pacífico Central de Costa Rica.

Resultados y conclusiones: Con base en estas experiencias, consideramos que estas tendencias metodológicas participativas tienen especial potencial para fortalecer estrategias 
de sostenibilidad, apoyado particularmente por el mayor acceso de la sociedad civil a tecnologías, el desarrollo de aplicaciones y herramientas tecnológicas de índole científico cada vez más fáciles de usar, así como una mayor conciencia ambiental a nivel mundial y específicamente en el contexto costarricense.

Palabras clave: Ciencia ciudadana, Sistemas de información geográfica participativos, Cuenca hidrográfica, Valores sociales, Calidad del agua

\begin{abstract}
Introduction and objective: Within the context of the II Research Sessions of the University of Costa Rica's (UCR) at the Faculty of Social Sciences, this article examines the social change potential of participatory methodologies for environmental management, especially within the emerging field of socio-hydrology. To this end, it introduces two current research projects, striving to influence decision-making at various governance levels.

Methods: Both projects apply new participatory approaches characterized by emphasizing the technological component. One of these projects relies on citizen science to strengthen environmental management capacities of local community groups through participatory monitoring of surface water quality in a watershed in the North Region of Costa Rica. This region is characterized by increasing social-ecological problems associated to water pollution from intensive agricultural activities. The other project presented utilizes participatory geographic information systems (PGIS) for mapping and spatially analyzing social values attributed to the ecosystem services provided by the Savegre fluvial system in the Central Pacific slope of Costa Rica.

Results and conclusions: Based on these experiences, it is considered that these methodological approaches have the potential to strengthen regional and local sustainability strategies. However, they must be supported with a greater access for civil society to technologies, an increased development of more user-friendly scientific applications and technological tools, as well as a greater environmental awareness, especially within the Costa Rican context.
\end{abstract}

Keywords: Citizen Science, Participatory geographic information systems, Watershed, Social values, Water quality. 


\section{Introducción}

Cada vez más, las Ciencias Sociales toman ventaja de los adelantos tecnológicos para incrementar la incidencia pública en los procesos de investigación científica enfocados en la toma de decisiones. Líneas de investigación en disciplinas que tratan con las interacciones sociedad-naturaleza como la Geografía, por ejemplo, buscan poner la tecnología científica al servicio de la ciudadanía para resoluciones importantes, esto como parte de un proceso de democratización de la ciencia que subyace en la toma de decisiones. Lo anterior ha sido particularmente notorio en estudios que vinculan el agua con procesos sociales, como en el campo multidisciplinario de la socio-hidrología (Sivapalan, Savenije, y Blöschl 2012). En este campo se ha visto un incremento exponencial de estudios que buscan involucrar más a la gente en decisiones asociadas al manejo del recurso hídrico. La tecnología de los sistemas de información geográfica (SIG) también ha sido cada vez más utilizada para incorporar a personas con poca formación técnico-científica en las decisiones con importantes dimensiones geoespaciales.

En este artículo, queremos examinar el potencial de estas tendencias metodológicas para la incidencia social, con el propósito de impulsar su aplicación en procesos de toma de decisiones en contextos con importantes retos vinculados a la gobernanza del agua. Creemos que investigaciones que contemplen estos abordajes metodológicos participativos pueden ayudar a robustecer la interfase ciencia-sociedad especialmente en Latinoamérica, una región caracterizada por la centralización en la toma de decisiones, corrupción, vulnerabilidad ante eventos hidrometereológicos extremos y a férreas oposiciones al manejo privado del agua (Assies 2003; Budds y McGranahan 2003). A nivel latinoamericano, cada vez más se promueven iniciativas para fortalecer el manejo de recursos naturales «de abajo hacia arriba», especialmente en temas del recurso hídrico (Delgado-Serrano et al. 2017).

Como medio para este objetivo, reflexionamos sobre algunas experiencias aprendidas hasta el momento en dos estudios de investigación actualmente en desarrollo en dos cuencas hidrográficas en Costa Rica, y que trabajan tendencias de investigación participativa en la 
socio-hidrología. Uno de esos estudios es sobre ciencia ciudadana aplicada al monitoreo de la calidad del agua en la cuenca del río Tres Amigos, en la Zona Norte de Costa Rica; mientras que el otro es un mapeo de valores sociales sobre servicios ecosistémicos asociados a los paisajes fluviales en la cuenca del río Savegre, en el Pacífico Central de Costa Rica.

Ciencia ciudadana: ¿promesa para democratizar la toma de decisiones a través de la ciencia?

\section{Reflexiones conceptuales sobre la ciencia ciudadana y su aplicación}

La ciencia ciudadana es una práctica que, si bien no es nueva en un sentido estricto, se ha popularizado mucho recientemente en campos disciplinares que integran lo social con lo ambiental. A pesar de que existen diversas conceptualizaciones de ciencia ciudadana, el común denominador es el componente participativo en procesos de índole científico de actores sin formación científica formal. Por otro lado, las divergencias metodológicas y conceptuales de ciencia ciudadana tienen que ver con los niveles de participación de las personas, así como con consideraciones más filosóficas sobre lo que debería ser ciencia ciudadana.

El concepto en sí de ciencia ciudadana no ha sido fácil de definir (Riesch y Potter 2014). A nivel general, su definición parece bifurcarse en dos nociones: la primera, y más criticada (Kimura y Kinchy 2016), alude a la simple colecta de datos por parte de los participantes. La segunda, y más compleja, la concibe más como un ejercicio de democratización de la ciencia con implicaciones sociales normativas. Adicionalmente, algunos conceptos asociados de la literatura en inglés (idioma en el que más se ha publicado sobre ciencia ciudadana), se suelen confundir con la ciencia ciudadana, como crowdsourcing, community science (ciencia comunitaria), civic science (ciencia cívica), community-based monitoring (monitoreo basado en la comunidad), volunteered geographic information (información geográfica voluntaria), entre otros. Si bien no es el objetivo de este artículo puntualizar sobre los debates conceptuales de ciencia ciudadana, coincidiremos con Hecker et al. $(2018,1)$ en que esta diversidad de conceptos hace «una comunidad de ciencia ciudadana, global y en crecimiento, dedicada a trabajar junta para tender un puente entre la interfaz ciencia-sociedad-política» 
(the result is a growing, global, citizen science community devoted to working together to bridge the science-society-policy interface).

Para mayor claridad, aquí definimos ciencia ciudadana como un proceso participativo en el cual las personas, sin entrenamiento científico formal, se involucran de forma activa en el desarrollo de un proceso científico-investigativo con un propósito normativo. Es una práctica que implica la participación de actores no-expertos a lo largo del proceso de investigación, desde el diseño inicial, hasta la implementación y fiscalización de las normativas resultantes. La ciencia ciudadana supone además una relación horizontal entre científicos y nocientíficos, la cual permite la construcción conjunta de conocimiento útil para la toma de decisiones.

Varios factores han sido claves en propiciar la popularidad reciente de la ciencia ciudadana. Por un lado, ha habido un vertiginoso desarrollo de las tecnologías de la información. En particular destaca un mayor acceso del público a internet, además de mejores y más rápidas conexiones, el uso ubicuo de teléfonos inteligentes y el creciente desarrollo de aplicaciones web y móviles para la manipulación y análisis de datos científicos (McQuillan 2014; Koch y Stisen 2017). Por otro lado, los centros de investigación, las ONG, entidades públicas, organizaciones comunitarias y la Academia deben cada vez más procurar «hacer más con menos», lo que ha llevado a diseñar procesos investigativos que minimicen costos y complicaciones logísticas (Kimura y Kinchy 2016; Pocock et al. 2017).

A nivel mundial, la ciencia ciudadana ha sido aplicada especialmente en temas ambientales, en particular al monitoreo de recursos naturales y biodiversidad (Chandler et al. 2017; McKinley et al. 2017). Recientemente, numerosas investigaciones y proyectos ambientales están apoyándose en procesos de ciencia ciudadana para mejorar las estrategias de conservación y manejo de recursos (Conrad y Hilchey 2011). En el mundo, particularmente en el área de socio-hidrología, la ciencia ciudadana es cada vez más popular; por ejemplo, para el monitoreo de la calidad del agua (p.ej. Shelton 2013; Jollymore et al. 2017) y de los niveles de corriente de los ríos (Lowry y Fienen 2013; Weeser et al. 2018), para el estudio de la distribución espacial de solutos de nitrógeno en aguas superficiales 
(Breuer et al. 2015), flujos de inundación en cuencas y áreas urbanas (Le Coz et al. 2016), modelación y caracterización de cuencas (Starkey et al. 2017) y para la evaluación de patrones espaciales en modelos hidrológicos (Koch y Stisen 2017), por nombrar solo algunas aplicaciones.

En Costa Rica, son relativamente pocos los proyectos de ciencia ciudadana que se han implementado. Muchos de los que se han llevado a cabo se han enfocado en el monitoreo de especies, donde destacan el Proyecto Aves y Ventanas de la Universidad Estatal a Distancia (UNED), la iniciativa sobre bioluminiscencia acuática del proyecto «Ciencia Ciudadana Bioluminiscencia Costa Rica», el monitoreo y seguimiento de bosques en recuperación de la Organización para Estudios Tropicales (OET) y la aplicación «¿Lo sentiste?» de la Red Sismológica Nacional de la Universidad de Costa Rica (RSN-UCR), entre otros. Existen otras iniciativas en el país que involucran la participación activa de ciudadanos en proyectos científicos, mas no explícitamente catalogadas como ciencia ciudadana. Además, en Costa Rica hay una marcada ausencia de experiencias de ciencia ciudadana relacionadas en el área de la hidrología.

\section{Potencial de incidencia social de la ciencia ciudadana}

Una pregunta central en discusiones sobre ciencia ciudadana es si tiene la capacidad para empoderar grupos sociales tradicionalmente marginados de procesos de toma de decisiones. Las respuestas, indudablemente, dependen del grado de involucramiento de los participantes a lo largo del proceso científico-normativo (p.ej, en la definición de objetivos y preguntas, elección de métodos, colecta de datos, análisis y evaluación de datos, comunicación de hallazgos y sistematización de lecciones aprendidas, diseño y fiscalización de normas) (Hecker et al. 2018). En efecto, las críticas más comunes a la ciencia ciudadana cuestionan esta visión de «extractivismo» de datos sin aspirar a un verdadero empoderamiento de la ciudadanía (Kimura y Kinchy 2016).

En efecto, conceptualizar la ciencia ciudadana como un proceso de democratización de la ciencia, más allá de la colecta de datos, implica que los participantes tengan «Voz y voto» 
desde el inicio hasta el fin del proceso científico-normativo (Capel 2014). Por ende, la ciencia ciudadana como práctica participativa es inherentemente política, y tiende a concebirse frecuentemente como una práctica de «contracultura»o «forma de resistencia» que atenta contra el autoritarismo científico de ciertos gremios de expertos y políticos (McQuillan 2014; Kullenberg 2015). Como lo señala Buytaert et al. (2014, 9): «el concepto de ciencia ciudadana, con el término "ciudadano" [...], sugiere una dimensión política - y no una dimensión técnica neutral» (The concept of citizen science with the term «citizen»... suggests a political — as opposed to a technical-neutral — dimension), que permite co-generar conocimiento accionable para la toma de decisiones, reduciendo el clasismo científico.

También, la ciencia ciudadana puede ser un mecanismo para facilitar espacios de colaboración en los que se reduzca la conflictividad en procesos de toma de decisiones. El diseñar procesos de toma de decisiones de abajo hacia arriba (bottom-up), en coordinación con niveles superiores de institucionalidad, puede facilitar consensos, así como forjar estructuras policéntricas que favorezcan la gobernanza ambiental efectiva (Ostrom 2009; Ostrom 2010). Además, los procesos de ciencia ciudadana que incorporen el conocimiento local/experiencial en los procesos científicos pueden ser determinantes para una gestión efectiva de recursos naturales (Gomez-Pompa y Kaus 1992). Con el involucramiento de participantes en el diseño de investigaciones científicas, la ciencia ciudadana promueve que los procesos de toma de decisiones se contextualicen adecuadamente, acorde con las realidades sociales y ambientales de las localidades y, con ello, se garantiza más el alcanzar los objetivos normativos. Finalmente, la ciencia ciudadana también puede ser un instrumento para la alfabetización y concientización científica fuera del ambiente formal académico, el cual puede ser inviable para muchas personas. Así, esta posibilidad puede fortalecer las capacidades técnico-científicas de miembros de la comunidad para una mejor toma de decisiones (Kimura y Kinchy 2016).

Sin duda, mucho del éxito de los procesos participativos en ciencia ciudadana depende en gran medida de las oportunidades que perciben los ciudadanos al conocer mejor su entorno e incidir efectivamente en las decisiones (Buytaert et al. 2014; Le Coz et al. 2016; Hecker et al. 2018). Mucho depende, no obstante, de la articulación entre los intereses de los científicos 
y los ciudadanos, así como del nivel de dependencia que los participantes tengan del recurso (Weeser et al. 2018).

\section{Proyecto de ciencia ciudadana en la cuenca del río Tres Amigos, Zona Norte}

El primer proyecto presentado en las II Jornadas de Investigación busca involucrar más activamente a las comunidades en la toma de decisiones a través del monitoreo de la calidad de las aguas superficiales en la cuenca del río Tres Amigos, en la Zona Norte de Costa Rica (Figura 1). Dicha investigación se titula «Ciencia ciudadana para la generación de información sobre calidad del agua en la cuenca del río Tres Amigos, en la Zona Norte de Costa Rica» y forma parte de las actividades del Observatorio del Agua y Cambio Global (OACG) de la Universidad de Costa Rica, concretamente el proyecto de investigación CHILD (por sus siglas en inglés: Costa Rica - Hydrology Impacts of El Niño and Development). Al ser un trabajo actualmente en desarrollo, el propósito aquí es presentar algunos detalles del proceso de esta investigación hasta el momento. 


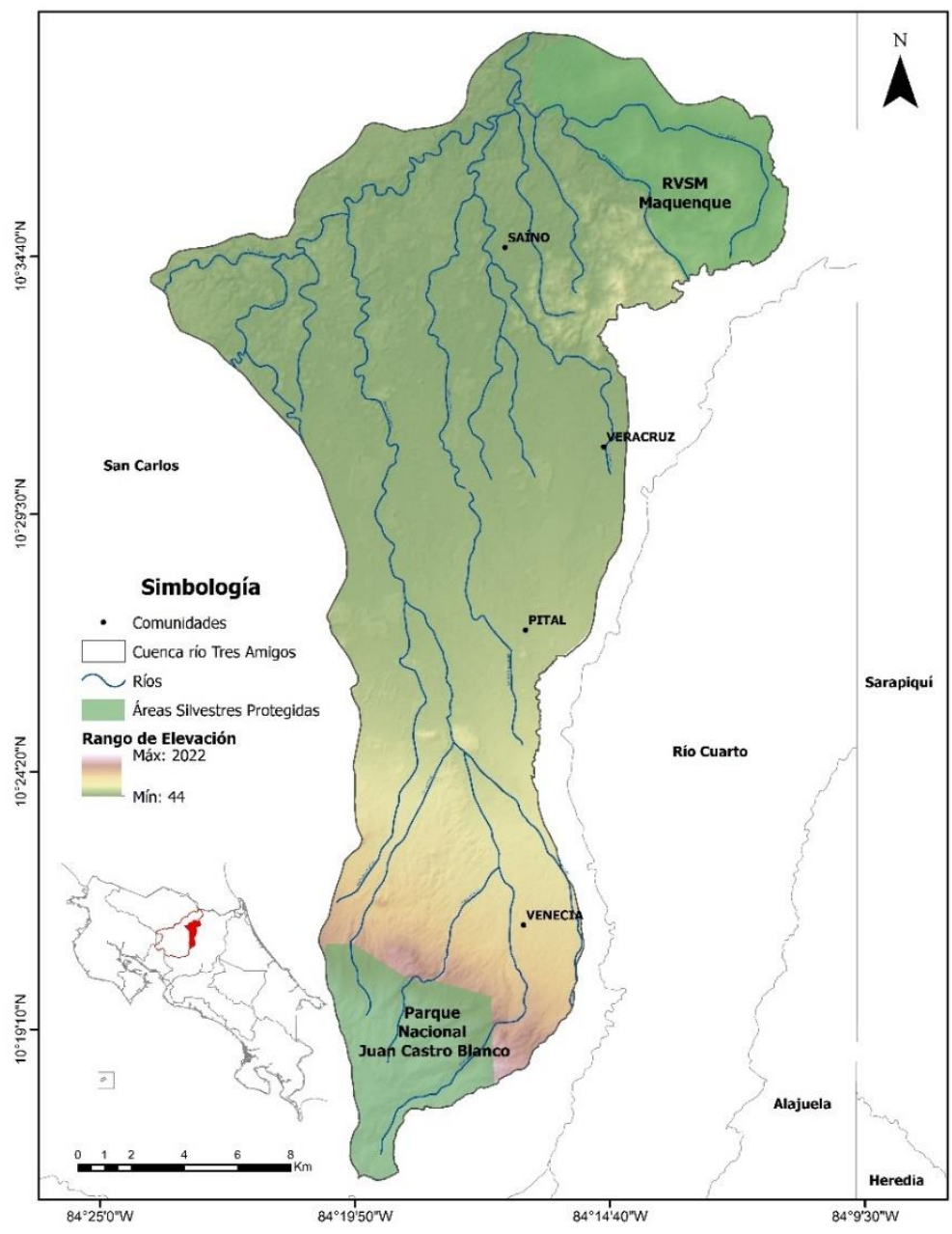

Figura 1. Zona de estudio del proyecto «Ciencia ciudadana para la generación de información sobre calidad del agua en la cuenca del río Tres Amigos, en la Zona Norte de Costa Rica».

Fuente: Elaboración propia.

Para desarrollar el proceso de ciencia ciudadana, este proyecto combina equipos de medición de calidad del agua superficial con una aplicación móvil capaz del mapeo y visualización de datos en tiempo real (Figura 2). Cada participante utiliza un equipo de bajo costo para la medición de ciertos parámetros de calidad del agua llamado Earth Force Low Cost Water Monitoring Kit. En el proceso participan administradores y fontaneros de las Asociaciones Administradoras de los Sistemas de Acueductos y Alcantarillados Comunales (ASADAS) de Venecia y Veracruz de Pital de San Carlos, administradores y miembros de

\section{(cc) BY-NC-ND}

Esta obra está bajo una Licencia Creative Commons Atribución-NoComercial-SinDerivadas 4.0 Internacional. 
la junta directiva de las Asociaciones de Desarrollo Integral (ADI), miembros de organizaciones ambientalistas y personas voluntarias de las comunidades, principalmente dueños de fincas ganaderas. Mediante talleres y pruebas de equipo en campo, cada participante fue debidamente capacitado tanto en el uso del equipo de monitoreo como de la aplicación móvil. También se realizaron dos talleres en el mes de febrero del 2019 para compartir experiencias, socializar resultados obtenidos al momento, recibir realimentación, así como para deliberar conjuntamente sobre posibles usos que se le puedan dar a los datos generados.

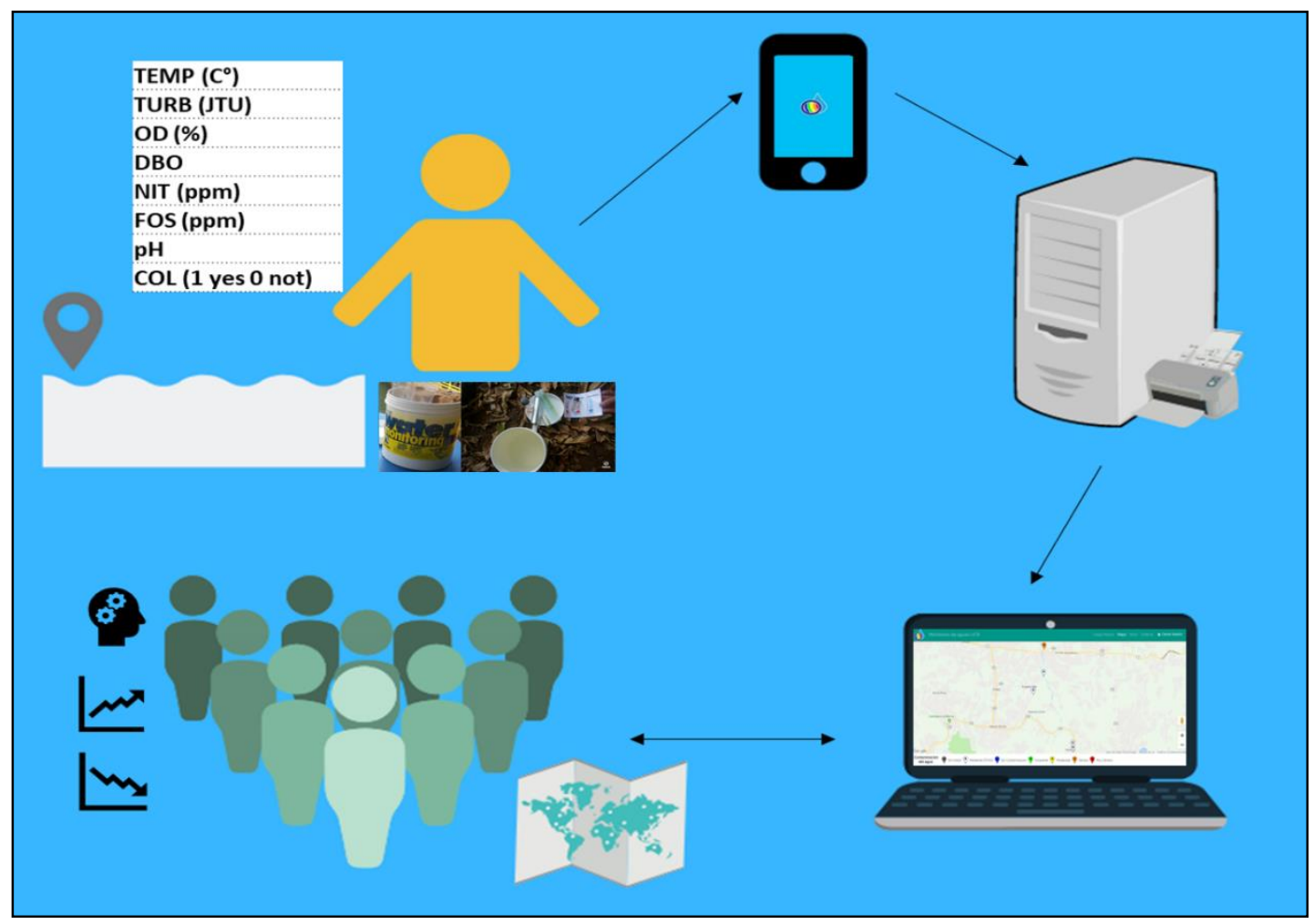

Figura 2. Esquema de flujo de trabajo que desarrolla el proyecto «Ciencia Ciudadana para la generación de información sobre calidad del agua en la cuenca del río Tres Amigos, en la Zona Norte de Costa Rica». El flujo inicia con la colecta de los datos en campo, luego el ingreso y registro de los datos en la aplicación móvil, la visualización de estos en la aplicación web, el análisis de información derivada en conjunto con los participantes y, finalmente, la socialización con otros actores locales.

Fuente: Alicia Correa del Observatorio del Agua y Cambio Global (OACG), Universidad de Costa Rica. Permiso obtenido.

\section{(cc) BY-NC-ND}

Esta obra está bajo una Licencia Creative Commons Atribución-NoComercial-SinDerivadas 4.0 Internacional. 
Los parámetros que se cuantifican con los equipos de monitoreo incluyen la presencia de coliformes fecales, temperatura, oxígeno disuelto, demanda bioquímica de oxígeno, $\mathrm{pH}$, nitratos y fosfatos (Figura 2). La recolección de datos se inició en diciembre del 2018, en diez puntos distribuidos a lo largo de la cuenca y con una frecuencia de monitoreo mensual. Para la validación de los resultados obtenidos de los equipos de monitoreo, se utiliza una sonda multiparamétrica Hanna Hi9828. Hasta el momento, esta validación ha constatado que los datos proporcionados por los participantes no presentan un margen de error amplio comparado con los de mayor precisión y que, por lo tanto, son confiables. A su vez, estas comparaciones de resultados también han sido compartidas y expuestas en los talleres y reuniones llevadas a cabo hasta el momento.

Los datos sobre calidad del agua recolectados por cada participante luego son ingresados, por ellos mismos, a una aplicación móvil para los sistemas operativos Android de Google ${ }$ llamada «Monitoreo de Agua» (Vázquez-Morera 2019), la cual permite registrar, visualizar, compartir y comparar los datos obtenidos. La aplicación además permite la visualización espacial de los datos utilizando la plataforma Google Maps, y cuenta con un componente web en el cual es posible hacer gráficas o series de tiempo para observar el comportamiento temporal de los datos.

Luego de los talleres efectuados, así como de las observaciones y entrevistas de campo, hemos identificado algunos resultados preliminares y lecciones aprendidas hasta el momento. Primero, los resultados preliminares revelan patrones similares y con un margen de error aceptable entre las mediciones hechas por los participantes y las efectuadas a través de la sonda multiparamétrica. Analizarlos de forma comparativa es trascendental para validar la calidad de los datos tomados por los participantes, y así aumentar la confianza por parte de los tomadores de decisiones. Por otro lado, y como era de esperar, el análisis de los datos obtenidos hasta ahora revela una significativa variación espacial y temporal de los parámetros monitoreados. Aquí rescatamos particularmente los nitratos y fosfatos, los cuales se asocian estrechamente con actividades productivas en la cuenca estudiada y son, por ende, de particular interés para los tomadores de decisiones.

\section{(cc) BY-NC-ND}

Esta obra está bajo una Licencia Creative Commons Atribución-NoComercial-SinDerivadas 4.0 Internacional. 
En general, las evaluaciones sobre el proceso de ciencia ciudadana hechas hasta ahora por los participantes han revelado criterios mayormente positivos. La mayoría ve la iniciativa con buenos grados de importancia, y todos los participantes han expresado deseos de continuar con el monitoreo por su cuenta una vez que concluya el proyecto. Asimismo, se ha resaltado la facilidad de utilizar el kit de monitoreo, así como la aplicación. Un aporte importante del proyecto, según los participantes consultados, ha sido el fortalecimiento de las redes de cooperación entre personas de las distintas comunidades involucradas, específicamente del sector de la cuenca media-baja. Muchos participantes perciben esta iniciativa como un mecanismo efectivo para coordinar acciones y fortalecer las redes de cooperación intercomunitarias ante problemáticas socio-ecológicas vinculadas a los ríos.

En cuanto a las lecciones aprendidas, notamos que la participación de los líderes comunitarios en el proceso ha mejorado la comprensión pública de la ciencia, y esto ha sido útil para cubrir vacíos de conocimiento en cuanto a la calidad del recurso hídrico en el contexto socioambiental de la cuenca. La ciencia ciudadana, en este caso, se percibe más por los participantes como una «vigilancia vecinal», que brinda la posibilidad de identificar contaminadores de una manera ordenada, sistemática y apoyada tecnológicamente; y que servirá para concientizar no solo a los actores locales, sino también a las autoridades tanto municipales como nacionales del problema socioambiental que se vive en estas comunidades. Asimismo, hemos notado que los niveles de compromiso y acción de los participantes están muy asociados con percepciones de las realidades socioambientales en los diferentes sectores de la cuenca. Por ejemplo, la contaminación hídrica por fincas piñeras que caracteriza al sector de la cuenca baja favorece la participación de un mayor número de personas en este sector preocupadas por el daño social y ambiental; en contraste, se nota un menor involucramiento en la cuenca alta, donde se asocian percepciones de agua limpia y abundante. Sin embargo, consideramos fundamental que estos procesos participativos fomenten una visión de la interdependencia funcional inherente en una cuenca hidrográfica entre los participantes y, con ello, ayudar a articular acciones a lo largo de todo el sistema de la cuenca. 


\section{Las tecnologías SIG como herramienta para fortalecer procesos participativos en la} toma de decisiones ambientales

\section{Concepto y aplicaciones socioambientales de los Sistemas de Información Geográfica Participativos (SIGP)}

Es innegable que los Sistemas de Información Geográfica (SIG) han transformado la ciencia geográfica, especialmente en mejorar los métodos y técnicas de cartografía y análisis espacial. Parte de la evolución que han tenido los SIG en las últimas décadas ha sido dejar de ser sistemas de uso exclusivo de técnicos y expertos, a volverse procesos más inclusivos en su uso para la toma de decisiones (Sieber 2006). De hecho, los Sistemas de Información Geográfica Participativos (de ahora en adelante SIGP) surgen en respuesta a peticiones para utilizar el SIG como medio para una mayor presencia pública en los procesos de toma de decisiones en diversos ámbitos y a múltiples escalas (Barrera Lobatón 2009). El término de SIGP $^{1}$ data de los años noventa, y alude al uso de tecnologías SIG para promover la participación pública en la colecta de datos, mapeo, análisis e interpretación de información geoespacial de diversa índole y para diversos propósitos. Como corolario al SIGP, usualmente se asocian el desarrollo de identidad comunitaria, construcción de capital social, y empoderamiento local (Chambers 2006; Brown y Kyttä 2014). De hecho, el SIGP ha sido frecuentemente utilizado por organizaciones comunitarias de desarrollo, las ONG, y grupos grassroots para incidencia social y ambiental.

Los SIGP han surgido como tecnología promisoria para lograr una mayor representatividad e inclusión social en la toma de decisiones con dimensiones geográficas. Sin embargo, han sido criticados especialmente por la calidad y confiabilidad de los datos geoespaciales, lo cual tiene ingentes implicaciones para su uso en procesos normativos y de planificación (Brown, Weber, y de Bie 2015). Muchas de estas consideraciones, no obstante, dependen de factores como la naturaleza de los datos, el diseño del proceso participativo, así como del propósito para el cual se buscan datos SIGP. Por ejemplo, con tal de procurar una

\footnotetext{
${ }^{1}$ Si bien se han debatido las diferencias y similitudes entre SIGP y otros términos asociados como SIG público participativo (PPGIS en inglés) e información geográfica voluntaria (VGI en inglés), en este artículo utilizaremos SIGP como concepto genérico. Para más detalle sobre estas discusiones, ver Brown y Kyttä (2014).
} 
mejor calidad de los datos, en el mapeo y análisis espacial de fenómenos físicos (como la distribución espacial de ecosistemas, especies, corrientes marinas, o formaciones geológicas), por lo general es preferible el criterio de expertos.

Pero para consideraciones más de índole sociocultural, como aceptación de estrategias de manejo o apoyo comunitario en procesos de gestión de recursos, un ejercicio de SIGP puede ser de mucha utilidad. En efecto, los SIGP fueron concebidos inicialmente para representar perspectivas subjetivas sobre el espacio geográfico y sus componentes. En muchos casos, lo que se pretende a través de ejercicios de mapeo participativo es justamente capturar subjetividades con el fin de incluirlas en la toma de decisiones, muchas veces para contrarrestar estructuras dominantes de poder que marginalizan sectores de la población y su acceso a recursos (Peluso 1995).

En ciertas situaciones, la inclusión de participantes es más bien ideal para mejorar la calidad de los datos. Por ejemplo, cuando los residentes de un sitio hacen el mapeo de sus entornos más inmediatos, pueden aportar sustancialmente al proceso gracias al íntimo conocimiento que tienen de su espacio (Heipke 2010; Werts et al. 2012). También se pueden obtener resultados particularmente útiles al combinar consideraciones biofísicas y sociales en el mismo ejercicio SIGP (p.ej. Whitehead et al. 2014), así como los criterios de expertos e inexpertos. Avances en las interacciones humano-computadora (HCI, en inglés) aplicado al SIGP, también pueden contribuir a mejorar la calidad de los datos obtenidos a través de una mejor visualización y representación de información geoespacial (Haklay y Tobón 2003).

Muchas aplicaciones de SIGP han sido para el estudio de componentes específicos de las interacciones sociedad-naturaleza, especialmente para optimizar la toma de decisiones en el manejo de recursos naturales. En el campo socio-hidrológico, los SIGP se han diseñado principalmente para procesos de soporte de decisiones en temas de administración del recurso hídrico (Nyerges et al. 2003), específicamente en conservación (Werts et al. 2012), monitoreo y análisis de calidad del agua (Bacic, Rossiter, y Bregt 2006; Luchette y Crawford 2008; Behmel et al. 2016), identificación de servicios ecosistémicos y sus valores sociales a nivel 
de cuenca (Cox et al. 2015; Lin et al. 2017), accesibilidad a ambientes acuáticos (Laatikainen et al. 2015), entre otros. Una de las aplicaciones más populares en este campo ha sido en el mapeo colaborativo de valores sociales atribuidos a los servicios ecosistémicos. A continuación, nos enfocamos en esta última aplicación, introduciendo primero los valores sociales aplicados a servicios ecosistémicos.

\section{Servicios ecosistémicos, valores sociales y Sistemas de Información Geográfica Participativos (SIGP)}

En las últimas décadas, diversos esfuerzos multidisciplinarios han buscado resaltar el vínculo entre naturaleza y bienestar humano en círculos científicos (MEA 2005; Balvanera et al. 2011). A través de proposiciones conceptuales-analíticas y metodológicas, el paradigma de los servicios ecosistémicos ha buscado estrechar esa conexión que, si bien se reconocía ampliamente, se tendía a omitir en evaluaciones ambientales y otros mecanismos de gestión ambiental. Los servicios ecosistémicos inicialmente surgen como un concepto a incluir en los cálculos económicos tradicionales que soslayaban la contribución del capital natural en la economía (Gómez-Baggethun et al. 2010). Con este enfoque, se buscó ampliar la gama de valores tanto monetarios como no monetarios que se le atribuían a la naturaleza, con el fin de darle mayor visibilidad a la gama de contribuciones de los ecosistemas en círculos principalmente políticos y económicos (Laurila-Pant et al. 2015; Jacobs et al. 2016). Indudablemente, a través de este paradigma se han profundizado las discusiones filosóficas sobre el abanico de valores atribuidos a la naturaleza (Kumar y Kumar 2008).

Desafortunadamente, este reconocimiento de la pluralidad valorativa de los servicios ecosistémicos ha tenido una tenue y lenta incidencia en procesos de evaluación ambiental (Ives y Kendal 2014; Jacobs et al. 2016). Esto ha sido especialmente conspicuo en el caso de los valores sociales, entendidos en este contexto como todos aquellos valores percibidos, no monetarios, que se atribuyen a los ecosistemas y los servicios que estos proveen (Sherrouse, Clement, y Semmens 2011). La consideración de este tipo de valores no es trivial, ya que representan una parte importante de los esquemas de valoración personales y colectivos que influyen en las decisiones ambientales. Considerarlos también permite evaluaciones 
ambientales más exhaustivas y realistas, que van más allá de los factores económicos. La Figura 3 muestra la relación entre la dinámica ecosistémica, la provisión de servicios para bienestar humano, loas diversas categorías de valores y la toma de decisiones derivada de esos esquemas.

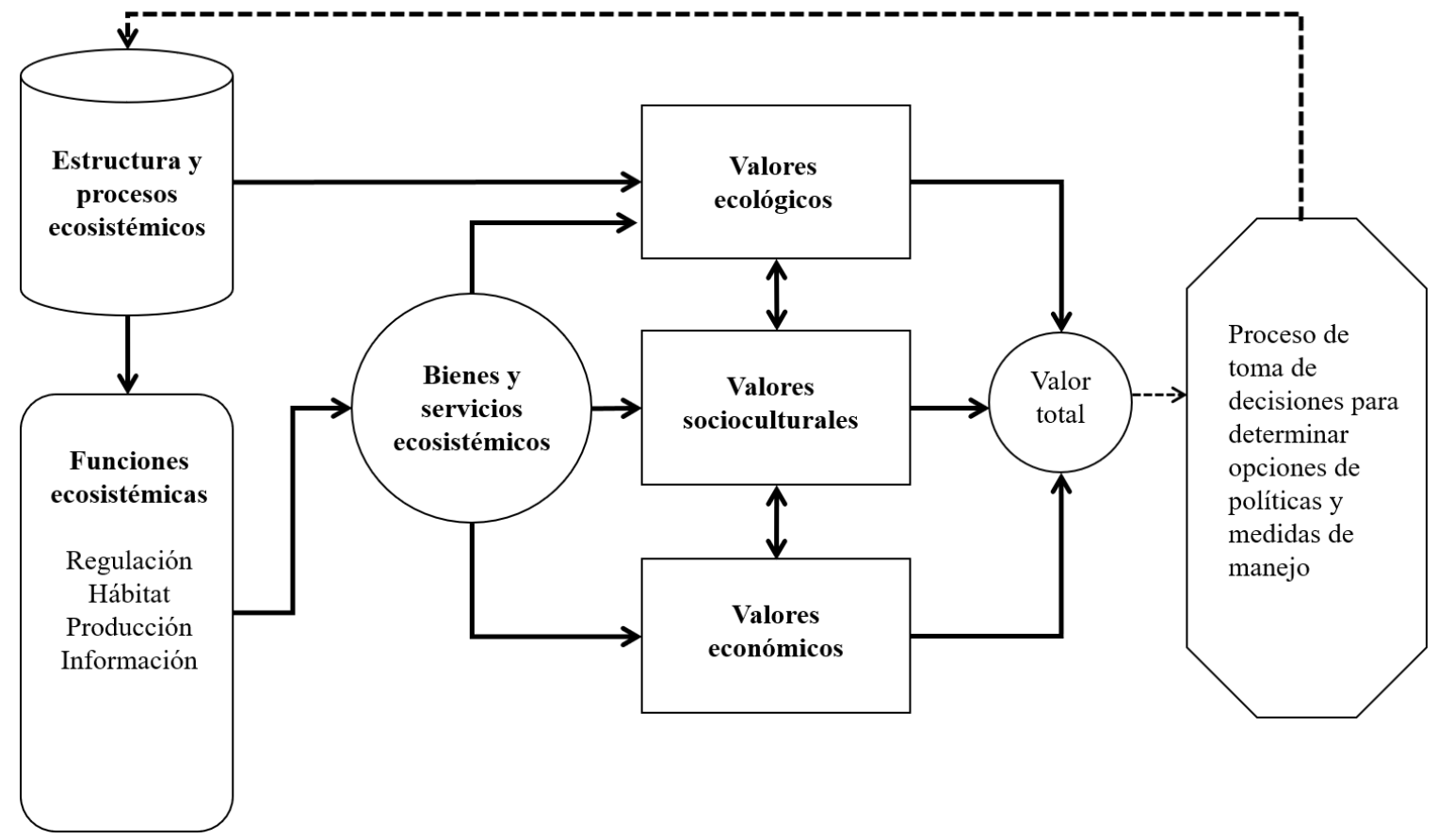

Figura 3. Relaciones entre ecosistemas, sus bienes y servicios derivados, diversos tipos de valores atribuidos a esos bienes y servicios, y la toma de decisiones resultante.

Fuente: Adaptado de De Groot et al. (2002). Traducción propia.

Como dijimos, una de las aplicaciones más populares de los SIGP ha sido para el mapeo de valores sociales de servicios ecosistémicos, en muchos casos con el propósito de incorporar criterios y valoraciones sobre servicios ecosistémicos a las evaluaciones ambientales. Por ejemplo, con el propósito de proveer insumos tradicionalmente omitidos en la toma de decisiones, los SIGP se han utilizado para el análisis de las dinámicas espaciales entre diferentes tipos de valores asociados a los servicios ecosistémicos (Van Riper y Kyle 2014), así como para examinar sinergias y diferencias entre percepciones subjetivas y cálculos más objetivos sobre servicios ecosistémicos (De Vreese et al. 2016; Bagstad et al. 2017).

\section{(c) BY-NC-ND}

Esta obra está bajo una Licencia Creative Commons Atribución-NoComercial-SinDerivadas 4.0 Internacional. 
Una herramienta de SIGP que se ha utilizado comúnmente para el mapeo, cuantificación y análisis de los valores sociales atribuidos a los servicios ecosistémicos es SolVES (en inglés: Social Values for Ecosystem Services o Valores Sociales para los Servicios Ecosistémicos; solves.cr.usgs.gov) (Sherrouse, Clement y Semmens 2011). SolVES se ideó con el fin de lograr una mayor incorporación de valoraciones sociales espacialmente explícitas a evaluaciones ambientales. Funciona como un «Add-in» en la barra de herramientas del programa ArcGIS de ESRI, y utiliza una serie de variables tanto ambientales (elevación, distancia a cuerpos de agua, entre otras) como sociales (preferencias, actitudes, entre otras) para calcular un índice de valoración social, representarlo espacialmente y realizar modelaciones sobre las relaciones entre variables. Gran parte de las investigaciones que han utilizado SolVES han buscado servir como insumo para la planificación de áreas protegidas y de recreación (Van Riper et al. 2012; Sherrouse, Semmens, y Clement 2014; Bagstad et al. 2017; Johnson et al. 2019), e incluso de parques urbanos (Sun et al. 2019).

\section{Proyecto de mapeo de valores sociales atribuidos a los paisajes fluviales en la cuenca del río Savegre}

El segundo proyecto sobre el cual presentamos avances en las II Jornadas de Investigación utilizó SIGP para el mapeo y análisis espacial de valores sociales atribuidos a los servicios ecosistémicos fluviales en la cuenca del río Savegre, Costa Rica (Figura 4). Esta cuenca fue designada en el año 2017 como parte de una Reserva de la Biosfera de la UNESCO, en gran parte por su riqueza biológica e importancia socioeconómica. Con un área de casi $600 \mathrm{~km}^{2}$, la cuenca presenta un pronunciado gradiente de elevación que se extiende desde los cero hasta aproximadamente los 3.400 metros sobre el nivel del mar, y que propicia una gran diversidad de microclimas y ecosistemas (Acevedo et al. 2002).

Hay variados usos de la tierra a lo largo de la cuenca. En la parte alta predominan los usos agrícolas, recreación, turismo y áreas protegidas estatales y privadas. En la parte media predominan los cultivos de café y, en menor grado, ganadería y turismo; mientras que en la cuenca baja se encuentran cultivos más intensificados como la palma africana y el arroz. Si bien en toda la cuenca hay una baja densidad poblacional con respecto a otras del país, esta 
ha estado sujeta a crecientes presiones antropogénicas por la expansión de las fronteras agrícolas y ganaderas, así como por actividades de acuicultura intensiva y pesca ilegal. Estas presiones contrastan con narrativas populares de cuenca poco alterada, de aguas limpias y de una elevada conciencia ambiental de sus pobladores (Gobierno de Costa Rica 2015). Esto último se refleja, por ejemplo, en movimientos intercomunitarios por evitar proyectos hidroeléctricos a gran escala, así como en el establecimiento de un robusto sector turístico basado en la naturaleza en las partes alta y media-baja de la cuenca.

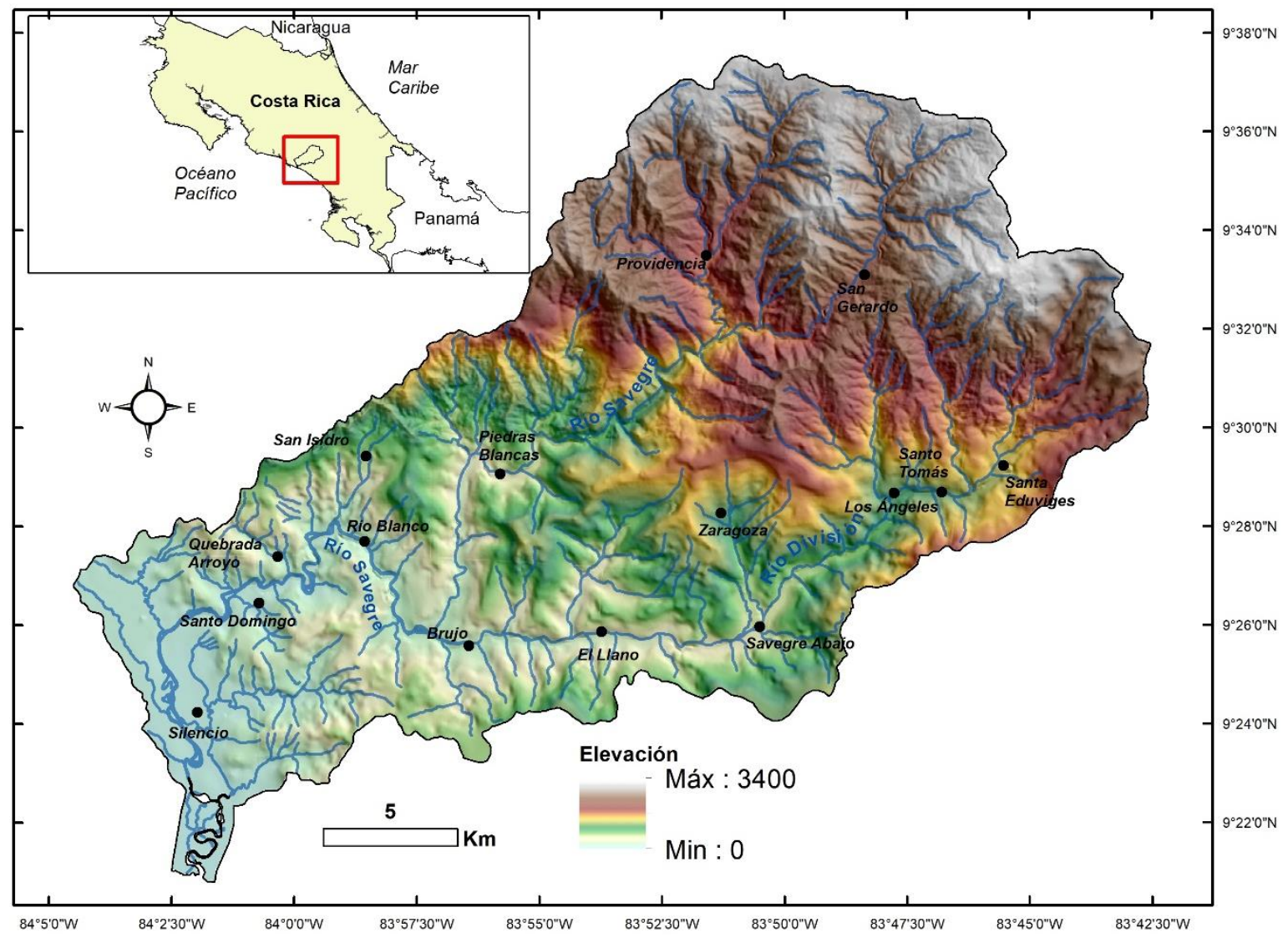

Figura 4. Mapa de la cuenca hidrográfica del río Savegre, con principales comunidades, red hídrica y gradiente altitudinal.

Fuente: Elaboración propia.

Sin embargo, para la cuenca del Savegre se carecen de insumos sobre preferencias y valoraciones sociales en torno a los servicios ecosistémicos que provee el sistema fluvial. Por

\section{(cc) BY-NC-ND}

Esta obra está bajo una Licencia Creative Commons Atribución-NoComercial-SinDerivadas 4.0 Internacional. 
eso, el proyecto en mención pretende capturar estas apreciaciones sociales en los administradores de las unidades productivas dentro de la cuenca usando SIGP, con el fin de incorporar estos criterios en evaluaciones ambientales que permitan diseñar mejores estrategias de sostenibilidad. Para este ejercicio, se utilizan mapas impresos que luego se procesan y analizan en un software SIG. También se utiliza la tipología de valores sociales especificada en Sherrouse, Clement y Semmens (2011) (Tabla 1),

Tabla 1. Valores sociales contemplados en el estudio en la cuenca del río Savegre presentado en las II Jornadas de Investigación de la Facultad de Ciencias Sociales, Universidad de Costa Rica

\begin{tabular}{|c|c|}
\hline Valor social & Descripción \\
\hline Valor estético & $\begin{array}{l}\text { Valoro este río porque disfruto del paisaje, de los sonidos, } \\
\text { vistas, aromas, etc. }\end{array}$ \\
\hline Valor de diversidad biológica & $\begin{array}{l}\text { Valoro este río porque provee una variedad de especies de } \\
\text { vida silvestre. }\end{array}$ \\
\hline Valor cultural & $\begin{array}{l}\text { Valoro este río porque representa parte de las tradiciones, } \\
\text { conocimiento y estilo de vida de mis ancestros. }\end{array}$ \\
\hline Valor económico & $\begin{array}{l}\text { Valoro este río porque provee de pescado, turismo, y agua } \\
\text { para uso humano. }\end{array}$ \\
\hline Valor futuro & $\begin{array}{l}\text { Valoro este río porque permite que futuras generaciones } \\
\text { conozcan y aprovechen el río a como está hoy. }\end{array}$ \\
\hline Valor histórico & $\begin{array}{l}\text { Valoro este río porque es importante para la historia natural } \\
\text { y humana de mi comunidad, región y país. }\end{array}$ \\
\hline Valor intrínseco & $\begin{array}{l}\text { Valoro este río solo porque existe, independientemente de la } \\
\text { utilidad que tenga a los humanos. }\end{array}$ \\
\hline Valor de Aprendizaje & $\begin{array}{l}\text { Valoro este río por su contribución a la educación, enseñanza } \\
\text { e investigación científica por parte de niños, jóvenes y } \\
\text { adultos. }\end{array}$ \\
\hline Valor de soporte de vida & $\begin{array}{l}\text { Valoro este río porque regula, preserva, limpia y renueva } \\
\text { agua y con ello la vida que dependen de ella. }\end{array}$ \\
\hline Valor de recreación & $\begin{array}{l}\text { Valoro este río porque me permite disfrutar de actividades } \\
\text { recreativas. }\end{array}$ \\
\hline Valor espiritual & $\begin{array}{l}\text { Valoro este río porque para mí tiene algún significado } \\
\text { espiritual o porque siento reverencia y respeto por él. }\end{array}$ \\
\hline Valor terapéutico & $\begin{array}{l}\text { Valoro este río porque me hace sentir mejor, física y } \\
\text { mentalmente. }\end{array}$ \\
\hline
\end{tabular}

Fuente: Adaptado y traducido de Sherrouse, Clement y Semmens (2011). 
Al igual que la investigación sobre ciencia ciudadana presentada anteriormente, este es un proyecto aún en desarrollo. Sin embargo, aquí sintetizamos algunos resultados preliminares y aprendizajes relevantes hasta el momento, en el contexto temático de este artículo. En cuanto a los valores sociales sobre servicios ecosistémicos, los resultados muestran una priorización de los valores de biodiversidad, futuro, estético, y soporte de vida (Figura 5), mientras que menos priorización se le dan a los valores intrínsecos y espirituales. Es importante resaltar la mayor priorización que se le da a valores de biodiversidad y futuros por sobre los tradicionalmente más priorizados, como el valor económico. En cuanto a las perspectivas espaciales, un resultado notable es que la mayoría de los participantes asocian ciertos valores con la unidad de la cuenca hidrográfica como un todo, o bien con una sección de ella, lo cual denota un entendimiento de la cuenca como un sistema funcionalmente interdependiente. Esta visión integrada predominó por sobre las atribuciones de valores sociales a niveles más desagregados (p.ej. a nivel de comunidad).

Lo anterior es importante para el diseño de estrategias de sostenibilidad a nivel de la cuenca, ya que apunta hacia una concepción articulada del sistema socio-ecológico y sus dinámicas. Por ejemplo, el hecho de que las comunidades de la parte alta de la cuenca atribuyan altos valores de biodiversidad a la parte media, puede favorecer la implementación de acciones en la cuenca alta que minimicen los impactos ambientales río abajo, sabiendo que hay una conexión funcional. De forma similar, el que los residentes de la cuenca baja otorguen un alto valor futuro a la cuenca alta por el rol de conservación de fuentes de agua, puede propiciar labores de cooperación entre sectores de la cuenca, como ha sucedido en el caso del Savegre. Asimismo, para priorizar las acciones de manejo de los ecosistemas de la cuenca, va a ser vital identificar las «zonas calientes» o hotspots de valores sociales a nivel de subcuenca, donde convergen muchos valores. 


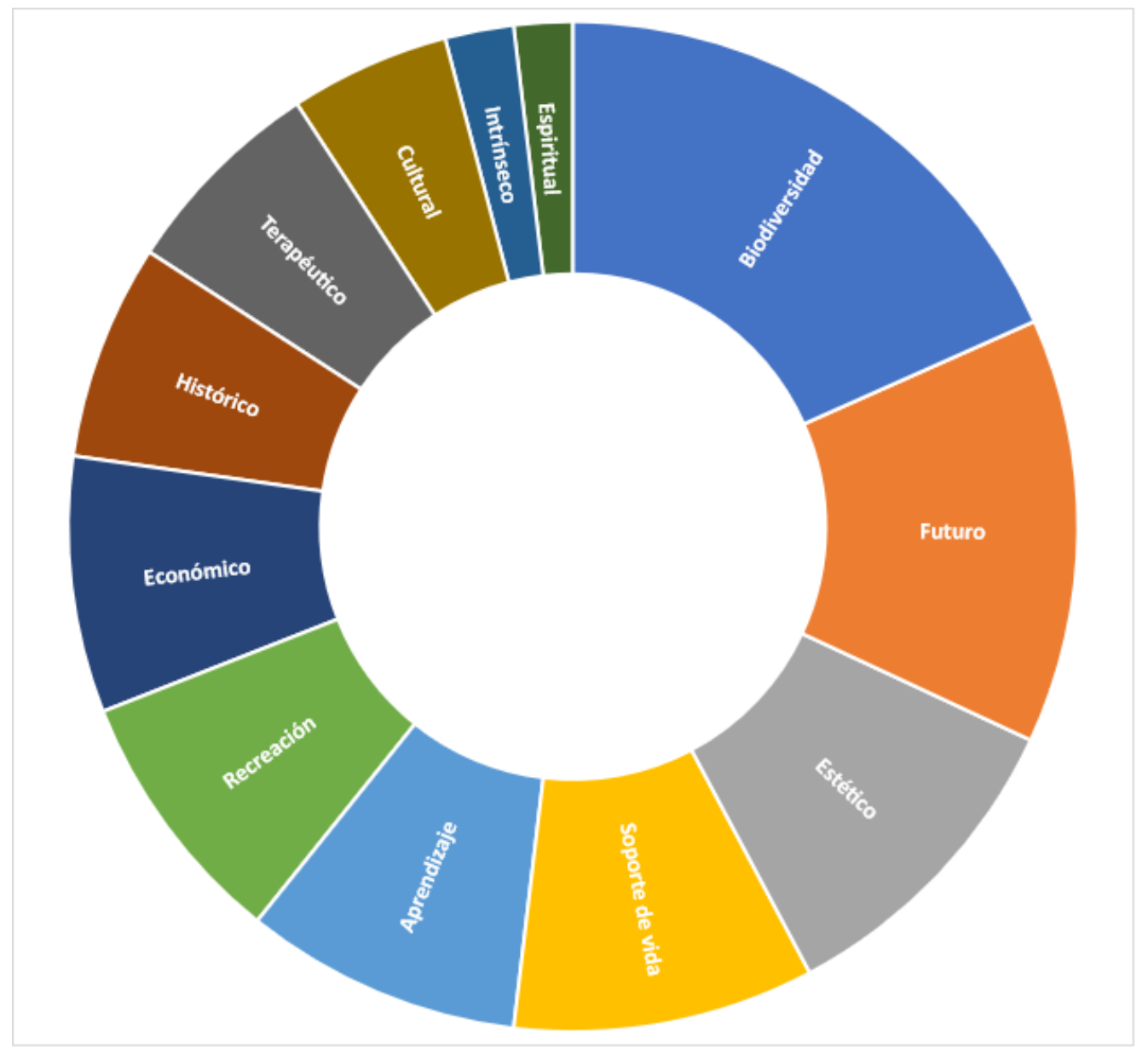

Figura 5. Diagrama que muestra niveles de priorización de valores sociales atribuidos a servicios ecosistémicos fluviales en la cuenca del Savegre basado en las dimensiones de cada categoría.

Fuente: Elaboración propia.

En cuanto a los aprendizajes del proceso, ha habido una notable anuencia de los participantes a trabajar con el concepto de valores sociales, esto porque amplía la gama de apreciaciones de la naturaleza más allá de consideraciones exclusivamente económicas. Percibimos que muchos participantes ya eran conscientes de esta diversidad (como en el caso de los valores de recreación, culturales y de diversidad biológica, por ejemplo; Tabla 1), mas desconocían su aplicabilidad a evaluaciones ambientales y, con ello, el potencial de incidencia. No obstante, este ejercicio SIGP también ha logrado sacar a la palestra pública valores más abstractos como el histórico, el espiritual, el de aprendizaje y el estético; los

\section{(cc) BY-NC-ND}

Esta obra está bajo una Licencia Creative Commons Atribución-NoComercial-SinDerivadas 4.0 Internacional. 
cuales usualmente no eran reconocidos por los participantes como valores que formalmente se le podían atribuir a los ecosistemas en evaluaciones ambientales.

En general, los participantes consideran que estas evaluaciones más integrales son trascendentales para apoyar procesos de toma de decisiones en materia de protección de recursos naturales y políticas públicas en el contexto de la cuenca. En particular, ha interesado el papel que puedan tener estos tipos de evaluaciones como insumos para los esquemas de incentivos, como los pagos por servicios ambientales (PSA). Esto es particularmente relevante en la cuenca del Savegre, donde ha habido problemas por el acceso a estos incentivos debido a disputas sobre tenencia de la tierra en áreas protegidas (SINAC 2017; Espinoza-Cisneros 2018).

También, la posibilidad de «espacializar» los valores sociales ha sido otro de los aspectos resaltados por los participantes. Para muchos, la noción de representar valores sociales en el espacio es novedoso y relevante para la toma de decisiones. Además, notamos que la interpretación de los valores más abstractos (p.ej. espiritual, terapéutico, futuro) se facilitó mucho con su representación en el espacio. Útil también fue la identificación de «puntos calientes» o hotspots, así como «puntos fríos» o coldspots, donde se concentran o desconcentran (respectivamente) los diferentes valores sociales.

Finalmente, en el contexto de un sistema funcionalmente interdependiente como una cuenca hidrográfica, para quienes toman decisiones en las comunidades puede ser muy provechoso el conocer las valoraciones que se hacen de otros sectores de la cuenca. En el caso de la cuenca del Savegre, si bien ha habido canales de comunicación y cooperación intercomunitarias, es necesario fortalecer estos vínculos y los procesos de planificación socio-ecológica para incrementar la resiliencia ante los retos socioambientales. En este aspecto, creemos que los ejercicios SIGP podrían contribuir significativamente.

\section{Discusión y conclusiones}

¿Pueden las nuevas tendencias en metodologías participativas lograr mayor incidencia social y robustecer los procesos de toma de decisiones en un contexto de

\section{(cc) BY-NC-ND}

Esta obra está bajo una Licencia Creative Commons Atribución-NoComercial-SinDerivadas 4.0 Internacional. 
apremiantes retos socioambientales? A continuación, tratamos con algunos aspectos que consideramos clave para discurrir respuestas a esta pregunta guía.

Ante crecientes retos socioambientales globales, y bajo la consigna de think global, act local (pensar global, actuar local), cada vez más se exige la democratización de la ciencia y descentralización en la toma de decisiones para la incidencia local/regional, pero con una visión global. Esto ha derivado en impulsar procesos de co-gestión adaptativa para proponer soluciones conjuntas y que involucren la participación de múltiples actores a diversas escalas, y a lo largo de todo el proceso de gestión ambiental, en un constante aprendizaje mutuo. Aunado a esto, cada vez se valora más la «contextualización de los procesos científicos» y se rehúye el aplicar «recetas universales» para la gestión ambiental, lo cual llama a actores locales a involucrarse en procesos de acción conjunta. Esto es aún más importante cuando se busca el empoderamiento e inclusión de grupos tradicionalmente marginados y subrepresentados en procesos de toma de decisiones para el manejo de recursos naturales.

Para lograr una mayor incidencia social y contribuir en el plano normativo, las corrientes disciplinarias que tratan con las interacciones ser humano-medio ambiente deben imperiosamente contemplar y apoyar estos avances en las metodologías participativas en el campo de la sostenibilidad, no solo en el ámbito de investigación, sino también en el de acción social. Por eso, desde la Academia hay que apoyar estos procesos, procurando su evolución hacia escenarios de mayor democratización, empoderamiento y, en general, hacia una toma de decisiones más efectiva. La Academia podría apoyar sustancialmente en la valoración conjunta de casos de aplicación, la sistematización de lecciones aprendidas, y determinar cómo adaptar mejor un proceso participativo a contextos particulares. Es vital también indagar más a fondo sobre las motivaciones detrás de la participación en estos procesos, así como las relaciones de poder que se dan y cómo estas podrían influir en el éxito o fracaso de un proceso.

También es indispensable nutrir una mayor voluntad política para utilizar la información resultante de los procesos de ciencia colectiva como insumo, especialmente en temas de manejo del agua. Este, sin duda, ha sido un tema sensible en círculos políticos ante 
la renuencia de los tomadores de decisiones a confiar en información científica co-generada entre expertos e inexpertos en asuntos de mucha trascendencia, como el manejo del agua o el uso de la tierra, por ejemplo. De hecho, una de las mayores críticas que se hacen a las metodologías participativas en general, es su poca incidencia probada en la toma de decisiones. Mucho de esto, sin embargo, consideramos que se relaciona con la falta de voluntad política para incorporar los insumos de estos procesos. Por eso, aquí es importante distinguir entre la poca incidencia probada (insistimos, mucho debido a la falta de voluntad política) y el potencial para incidir. Este potencial, creemos, se puede acentuar aún más en el contexto costarricense, caracterizado por una creciente conciencia ambiental de su población (Informe Estado de la Nación 2017). Evidentemente, para ello es trascendental garantizar la calidad de la información suministrada, pero con la tecnología esto puede mejorar sustancialmente.

Sin duda, las tecnologías han jugado un papel medular (sino el más importante) en esta evolución de las metodologías participativas en los últimos años al facilitar una mayor apertura en los procesos de toma de decisiones. Un mayor acceso a teléfonos inteligentes con aplicaciones cada vez más sofisticadas, pero fáciles de usar, se presta para un mayor involucramiento de la población, así como para la obtención de gran cantidad de datos en tiempo real para la toma de decisiones. Esto es aún más significativo para el caso del agua, cuyas dinámicas han sido ampliamente estudiadas y modeladas desde diversos ámbitos comparado a otros recursos (como la biodiversidad, por ejemplo), lo que permite integrar, apoyar y complementar los procesos participativos con bases de datos, mapas y modelos ya existentes. Estas ventajas se reflejan en el vertiginoso desarrollo en los últimos años de muchas aplicaciones web y móviles en temas de agua, que ayudan a manipular datos y realizar modelados de dinámicas hidrológicas por no-expertos. En el campo del SIGP, eso es igualmente promisorio, con el desarrollo de plataformas digitales de mapeo y visualización espacial cada vez más robustas y amigables al usuario como Google Maps, Google Earth, OpenStreetMap, etc., así como sofisticados programas SIG de código abierto como QGIS que permiten un mayor acceso a estas tecnologías. Ante esto, sin embargo, es vital que la tecnología no desvíe los procesos participativos del objetivo primordial de procurar el 
involucramiento efectivo en la toma de decisiones de sectores tradicionalmente subrepresentados (Brown y Kyttä 2014). Por eso, entre las preguntas importantes al diseñar estos procesos están quiénes deben participar, a través de qué tecnología, por qué y para qué (Tadaki, Sinner, y Chan 2017).

Queremos realzar la importancia de aplicar estas metodologías participativas para la toma de decisiones en temas del agua, por lejos el recurso natural más esencial, y además muy dependiente del contexto para su manejo efectivo. Aunado a esto, ante la incertidumbre socio-ecológica por el cambio climático, va a ser aún más importante un manejo colectivo y participativo del recurso hídrico para la gestión de riesgo ante sequías o escasez, contaminación, inundaciones, degradación de ecosistemas acuáticos, entre otras problemáticas asociadas al cambio global. En las últimas décadas se han discurrido esquemas participativos para el manejo del agua, como el Manejo Integrado del Recurso Hídrico (MIRH), que, en su esencia, buscan una mayor inclusión social en el manejo de este recurso. El agua es un recurso que tiende a generar tensiones si es mal manejado, por lo que el aplicar procesos que procuren mayor democratización y representación en procesos de toma de decisiones es esencial para el orden social.

Por último, el agua también es un recurso del cual se derivan múltiples servicios ecosistémicos más allá de la simple provisión de agua, y esta diversidad de servicios debe idealmente ser gestionada de manera abierta y participativa. Como dijimos, esas diversas valoraciones de los servicios proveídos por el agua deben ser incorporadas en instrumentos de evaluación ambiental, para procurar una toma de decisiones más consciente de la totalidad de costos y beneficios de una decisión más allá de lo meramente económico. Sin duda, el agua es un recurso alrededor del cual históricamente han convergido complejas relaciones de poder, así como múltiples significados y valoraciones. Por eso, el camino hacia la democratización y participación cívica en procesos de toma de decisiones, si bien puede ser escabroso, es indispensable para una gobernanza ambiental verdaderamente orientada hacia la sostenibilidad global. 
Contribución de autores: Édgar Espinoza Cisneros (EEC) contribuyó con la parte del SIGP y el caso de la cuenca del Savegre. Sara Blanco Ramírez (SBR) contribuyó con la parte de ciencia ciudadana y el caso de estudio en la cuenca del río Tres Amigos en San Carlos. Tanto EEC como SBR contribuyeron en la sección de conclusiones y la introducción. EEC realizó una revisión final exhaustiva de todo el documento para editarlo y mejorar su redacción.

Apoyo financiero: La investigación en la cuenca del río Tres Amigos tuvo el apoyo financiero del Espacio Universitario de Estudios Avanzados de la UCR (UCREA), bajo el proyecto B8276 - CHILD (Costa Rica - Hydrology Impacts of El Niño and Development). La investigación en la cuenca del río Savegre contó con el apoyo financiero de la Vicerrectoría de la Investigación de la UCR, bajo el proyecto B6355.

\section{Referencias}

Acevedo, Heiner, Julio Bustamante, Luis Paniagua, y Ronald Chaves. 2002. Ecosistemas de la Cuenca Hidrográfica Del Río Savegre, Costa Rica. Santo Domingo de Heredia: Editorial INBio.

Assies, Willem. 2003. "David versus Goliath in Cochabamba: Water Rights, Neoliberalism, and the Revival of Social Protest in Bolivia." Latin American Perspectives 30 (3): 1436.

Bacic, Ivan Luiz Zilli, David G Rossiter, y Arnold K Bregt. 2006. "Using Spatial Information to Improve Collective Understanding of Shared Environmental Problems at Watershed Level." Landscape and Urban Planning 77 (1-2): 54-66.

Bagstad, Kenneth J, Darius J Semmens, Zachary H Ancona, y Benson C Sherrouse. 2017. "Evaluating Alternative Methods for Biophysical and Cultural Ecosystem Services Hotspot Mapping in Natural Resource Planning." Landscape Ecology 32 (1): 77-97.

Balvanera, Patricia, Alicia Castillo, P Avila, Karina Caballero, A Flores, C Galicia, L M Galindo, et al. 2011. "Marcos Conceptuales Interdisciplinarios Para El Estudio de Los Servicios Ecosistémicos En América Latina." En Valoración de Servicios Ecosistémicos: Conceptos, Herramientas y Aplicaciones Para El Ordenamiento Territorial, editado por Pedro Laterra, Esteban Jobbágy, y José Paruelo, 38-67. Ediciones INTA.

Barrera Lobatón, Susana. 2009. "Reflexiones Sobre Sistemas de Información Geográfica Participativos (SIGP) y Cartografía Social.” Cuadernos de Geografía: Revista 
Colombiana de Geografía 18: 9-23.

Behmel, Sonja, Mathieu Damour, Ralf Ludwig, y Manuel Rodriguez. 2016. "Water Quality Monitoring Strategies - A Review and Future Perspectives." Science of the Total Environment 571: 1312-29.

Breuer, Lutz, Noreen Hiery, Philipp Kraft, Martin Bach, Alice H Aubert, y Hans-Georg Frede. 2015. "HydroCrowd: A Citizen Science Snapshot to Assess the Spatial Control of Nitrogen Solutes in Surface Waters." Scientific Reports 5: 16503.

Brown, Greg, y Marketta Kyttä. 2014. "Key Issues and Research Priorities for Public Participation GIS (PPGIS): A Synthesis Based on Empirical Research." Applied Geography 46: 122-36.

Brown, Greg, Delene Weber, y Kelly de Bie. 2015. "Is PPGIS Good Enough? An Empirical Evaluation of the Quality of PPGIS Crowd-Sourced Spatial Data for Conservation Planning." Land Use Policy 43: 228-38.

Budds, Jessica, y Gordon McGranahan. 2003. "Are the Debates on Water Privatization Missing the Point? Experiences from Africa, Asia and Latin America." Environment and Urbanization 15 (2): 87-114.

Buytaert, Wouter, Zed Zulkafli, Sam Grainger, Luis Acosta, Tilashwork C Alemie, Johan Bastiaensen, Bert De Bièvre, et al. 2014. "Citizen Science in Hydrology and Water Resources: Opportunities for Knowledge Generation, Ecosystem Service Management, and Sustainable Development." Frontiers in Earth Science 2: 26.

Capel, Horacio. 2014. “Ciencia Ciudadana, Ética y Política Para Viejos y Nuevos Problemas." Biblio 3W. Revista Bibliográfica de Geografía y Ciencias Sociales 19 (1096).

Chambers, Robert. 2006. "El Mapeo Participativo y Los Sistemas de Información Geográfica: ¿De Quién Son Los Mapas? ¿Quién Se Empodera y Quién Se Desempodera? ¿Quién Gana y Quién Pierde?” EJISDC 25 (2): 1-12.

Chandler, Mark, Linda See, Kyle Copas, Astrid M.Z. Bonde, Bernat Claramunt López, Finn Danielsen, Jan Kristoffer Legind, et al. 2017. "Contribution of Citizen Science towards International Biodiversity Monitoring." Biological Conservation 213: 280-94. https://doi.org/10.1016/j.biocon.2016.09.004.

Conrad, Cathy C, y Krista G Hilchey. 2011. "A Review of Citizen Science and CommunityBased Environmental Monitoring: Issues and Opportunities." Environmental Monitoring and Assessment 176 (1-4): 273-91.

\section{(cc) BY-NC-ND}

Esta obra está bajo una Licencia Creative Commons Atribución-NoComercial-SinDerivadas 4.0 Internacional. 
Cox, Cody, Wayde Morse, Christopher Anderson, y Luke Marzen. 2015. "Using Public Participation Geographic Information Systems to Identify Places of Watershed Service Provisioning." JAWRA Journal of the American Water Resources Association 51 (3): 704-18.

Coz, Jérôme Le, Antoine Patalano, Daniel Collins, Nicolás Federico Guillén, Carlos Marcelo García, Graeme M Smart, Jochen Bind, et al. 2016. "Crowdsourced Data for Flood Hydrology: Feedback from Recent Citizen Science Projects in Argentina, France and New Zealand.” Journal of Hydrology 541: 766-77.

Espinoza-Cisneros, Edgar. 2018. “Optimizing Social-Ecological Analysis of Coupled Human-River Systems through the Integration of Conceptual Frameworks: The Case of the Savegre Watershed, Costa Rica." Revista Geográfica de América Central 61E (3): $32-50$.

Gobierno de Costa Rica. 2015. "Gobierno Decretó Salvaguarda Ambiental de 25 Años Para Ríos Pacuare y Savegre." Comunicados de La Presidencia de La República de Costa

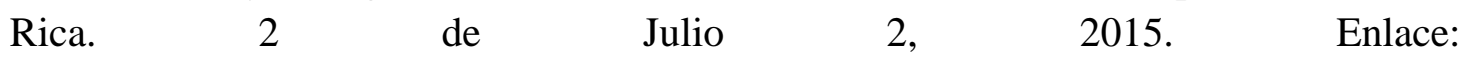
http://presidencia.go.cr/comunicados/2015/08/gobierno-decreto-salvaguardaambiental-de-25-anos-para-rios-pacuare-y-savegre-2/.

Gómez-Baggethun, Erik, Rudolf De Groot, Pedro L Lomas, y Carlos Montes. 2010. "The History of Ecosystem Services in Economic Theory and Practice: From Early Notions to Markets and Payment Schemes." Ecological Economics 69 (6): 1209-18.

Gomez-Pompa, Arturo y Andrea Kaus. 1992. "Taming the Wilderness Myth.” BioScience 42 (4): 271-79.

De Groot, Rudolf, Matthew A Wilson, y Roelof M J Boumans. 2002. "A Typology for the Classification, Description and Valuation of Ecosystem Functions, Goods and Services." Ecological Economics 41 (3): 393-408.

Haklay, Mordechai, y Carolina Tobón. 2003. "Usability Evaluation and PPGIS: Towards a User-Centred Design Approach.” International Journal of Geographical Information Science 17 (6): 577-92.

Hecker, Susanne, Rick Bonney, Muki Haklay, Franz Hölker, Heribert Hofer, Claudia Goebel, Margaret Gold, et al. 2018. "Innovation in Citizen Science--Perspectives on SciencePolicy Advances." Citizen Science: Theory and Practice 3 (1).

Heipke, Christian. 2010. "Crowdsourcing Geospatial Data." ISPRS Journal of Photogrammetry and Remote Sensing 65 (6): 550-57. 
Informe Estado de la Nación. 2017. "Hallazgos Iniciales de La Encuesta "Patrones y Percepciones Ciudadanas Sobre Medio Ambiente y Condiciones Para El Cambio". Aporte Especial. Capítulo 4. San José, Costa Rica: Programa Estado de La Nación.

Ives, Christopher D., y Dave Kendal. 2014. "The Role of Social Values in the Management of Ecological Systems." Journal of Environmental Management 144: 67-72.

Jacobs, Sander, Nicolas Dendoncker, Berta Martín-López, David Nicholas Barton, Erik Gomez-Baggethun, Fanny Boeraeve, Francesca L McGrath, et al. 2016. "A New Valuation School: Integrating Diverse Values of Nature in Resource and Land Use Decisions." Ecosystem Services 22: 213-20.

Johnson, Dana N, Carena J. van Riper, Maria Chu, y Sophia Winkler-Schor. 2019. "Comparing the Social Values of Ecosystem Services in US and Australian Marine Protected Areas." Ecosystem Services 37: 100919.

Jollymore, Ashlee, Morgan J Haines, Terre Satterfield, y Mark S Johnson. 2017. "Citizen Science for Water Quality Monitoring: Data Implications of Citizen Perspectives." Journal of Environmental Management 200: 456-67.

Kimura, Aya H, y Abby Kinchy. 2016. "Citizen Science: Probing the Virtues and Contexts of Participatory Research.” Engaging Science, Technology, and Society 2: 331-61.

Koch, Julian, y Simon Stisen. 2017. "Citizen Science: A New Perspective to Advance Spatial Pattern Evaluation in Hydrology.” PloS One 12 (5): e0178165.

Kullenberg, Christopher. 2015. "Citizen Science as Resistance: Crossing the Boundary between Reference and Representation.” Journal of Resistance Studies 1 (1): 50-76.

Kumar, Manasi, y Pushpam Kumar. 2008. "Valuation of the Ecosystem Services: A PsychoCultural Perspective.” Ecological Economics 64 (4): 808-19.

Laatikainen, Tiina, Henrikki Tenkanen, Marketta Kyttä, y Tuuli Toivonen. 2015. "Comparing Conventional and PPGIS Approaches in Measuring Equality of Access to Urban Aquatic Environments." Landscape and Urban Planning 144: 22-33.

Laurila-Pant, Mirka, Annukka Lehikoinen, Laura Uusitalo, y Riikka Venesjärvi. 2015. "How to Value Biodiversity in Environmental Management?" Ecological Indicators 55: 1-11.

Lin, Yu-Pin, Wei-Chih Lin, Hsin-Yi Li, Yung-Chieh Wang, Chih-Chen Hsu, Wan-Yu Lien, Johnathen Anthony y Joy R Petway. 2017. "Integrating Social Values and Ecosystem Services in Systematic Conservation Planning: A Case Study in Datuan Watershed." Sustainability 9 (5): 718.

\section{(cc) BY-NC-ND}

Esta obra está bajo una Licencia Creative Commons Atribución-NoComercial-SinDerivadas 4.0 Internacional. 
Lowry, Christopher S. y Michael N Fienen. 2013. "CrowdHydrology: Crowdsourcing Hydrologic Data and Engaging Citizen Scientists.” GroundWater 51 (1): 151-56.

Luchette, Joseph A, y Tom Crawford. 2008. "A Public Participation GIS Application for Citizen-Based Watershed Monitoring in the Pamlico-Tar River Basin, North Carolina." Southeastern Geographer 48 (2): 184-200.

McKinley, Duncan C., Abe J. Miller-Rushing, Heidi L. Ballard, Rick Bonney, Hutch Brown, Susan C. Cook-Patton, Daniel M. Evans, et al. 2017. "Citizen Science Can Improve Conservation Science, Natural Resource Management, and Environmental Protection." Biological Conservation 208: 15-28. s

McQuillan, Daniel. 2014. "The Countercultural Potential of Citizen Science." M/C Journal 17 (6).

MEA, (Millennium Ecosystem Assessment). 2005. Ecosystems and Human Well-Being: Current State and Trends. Millennium Ecosystem Assessment. Vol. 1. Washington, D.C.: Island Press.

Nyerges, Timothy L., Piotr Jankowski, Kevin Ramsey, y David Tuthill. 2003. "PPGIS in Collaborative Water Resource Decision Making: Results of a Field Experiment." In 2nd Annual Public Participation in GIS Conference: Portland, OR., E.E.U.U.

Ostrom, Elinor. 2009. A Polycentric Approach for Coping with Climate Change. World Bank Policy Research Working Paper 5095: The World Bank. https://ssrn.com/abstract=1494833

- 2010. "Polycentric Systems for Coping with Collective Action and Global Environmental Change." Global Environmental Change 20 (4): 550-57.

Peluso, Nancy Lee. 1995. "Whose Woods Are These? Counter-Mapping Forest Territories in Kalimantan, Indonesia." Antipode 27 (4): 383-406.

Pocock, Michael J O, John C Tweddle, Joanna Savage, Lucy D Robinson, y Helen E Roy. 2017. "The Diversity and Evolution of Ecological and Environmental Citizen Science." PloS One 12 (4): e0172579.

Riesch, Hauke, y Clive Potter. 2014. "Citizen Science as Seen by Scientists: Methodological, Epistemological and Ethical Dimensions." Public Understanding of Science 23 (1): 107-20.

Shelton, Ashley. 2013. "The Accuracy of Water Quality Monitoring Data: A Comparison between Citizen Scientists and Professionals." Saint Mary’s University, Halifax, Nova 
Scotia.

Sherrouse, Benson C, Jessica M Clement, y Darius J Semmens. 2011. "A GIS Application for Assessing, Mapping, and Quantifying the Social Values of Ecosystem Services." Applied Geography 31 (2): 748-60.

Sherrouse, Benson C, Darius J Semmens, y Jessica M Clement. 2014. "An Application of Social Values for Ecosystem Services (SolVES) to Three National Forests in Colorado and Wyoming." Ecological Indicators 36: 68-79.

Sieber, Renee. 2006. "Public Participation Geographic Information Systems: A Literature Review and Framework." Annals of the Association of American Geographers 96 (3): 491-507.

SINAC. 2017. "Sistematización Desarrollo Sostenible de La Cuenca Hidrográfica Del Río Savegre.” Puntarenas, Costa Rica: Sistema Nacional De Áreas de Conservación Ministerio de Ambiente y Energía.

Sivapalan, Murugesu, Hubert H G Savenije, y Günter Blöschl. 2012. "Socio-Hydrology: A New Science of People and Water." Hydrological Processes 26 (8): 1270-76.

Starkey, Eleanor, Geoff Parkin, Stephen Birkinshaw, Andy Large, Paul Quinn, y Ceri Gibson. 2017. "Demonstrating the Value of Community-Based ('Citizen Science') Observations for Catchment Modelling and Characterisation." Journal of Hydrology 548: 801-17.

Sun, Fengyun, Jingyi Xiang, Yun Tao, Chunfu Tong, y Yue Che. 2019. "Mapping the Social Values for Ecosystem Services in Urban Green Spaces: Integrating a Visitor-Employed Photography Method into SolVES." Urban Forestry \& Urban Greening 38: 105-13.

Tadaki, Marc, Jim Sinner, y Kai M.A. Chan. 2017. "Making Sense of Environmental Values: A Typology of Concepts." Ecology and Society 22 (1).

Van Riper, Carena J., y Gerard T. Kyle. 2014. "Capturing Multiple Values of Ecosystem Services Shaped by Environmental Worldviews: A Spatial Analysis." Journal of Environmental Management 145: 374-84.

Van Riper, Carena J., Gerard T Kyle, Stephen G Sutton, Melinda Barnes, y Benson C Sherrouse. 2012. "Mapping Outdoor Recreationists' Perceived Social Values for Ecosystem Services at Hinchinbrook Island National Park, Australia." Applied Geography 35 (1-2): 164-73.

Vásquez-Morera, Javier (2019). Monitoreo-Agua-UCR (Versión 1.057) [Software de

\section{(cc) BY-NC-ND}

Esta obra está bajo una Licencia Creative Commons Atribución-NoComercial-SinDerivadas 4.0 Internacional. 
aplicación

móvil].

Recuperado

de

https://drive.google.com/drive/folders/1s9xhBL7duG-t3yvvnOXiiZbDnYU11rK3

Vreese, Rik De, M Leys, C M Fontaine, y Nicolas Dendoncker. 2016. "Social Mapping of Perceived Ecosystem Services Supply--The Role of Social Landscape Metrics and Social Hotspots for Integrated Ecosystem Services Assessment, Landscape Planning and Management." Ecological Indicators 66: 517-33.

Weeser, Björn, Jaqueline Stenfert Kroese, Suzanne Jacobs, Naomi K. Njue, Zacchaeus Kemboi, Anne Marie Ran, Mariana C. Rufino, y Lutz Breuer. 2018. "Citizen Science Pioneers in Kenya--A Crowdsourced Approach for Hydrological Monitoring." Science of The Total Environment 631: 1590-99.

Werts, Joshua D, Elena A Mikhailova, Christopher J Post, y Julia L Sharp. 2012. “An Integrated WebGIS Framework for Volunteered Geographic Information and Social Media in Soil and Water Conservation." Environmental Management 49 (4): 816-32.

Whitehead, Amy L, Heini Kujala, Christopher D Ives, Ascelin Gordon, Pia E Lentini, Brendan A Wintle, Emily Nicholson, y Christopher M Raymond. 2014. "Integrating Biological and Social Values When Prioritizing Places for Biodiversity Conservation." Conservation Biology 28 (4): 992-1003. 